\title{
Efectividad de baclofeno en el tratamiento de espasticidad de origen cerebral
}

\author{
Isabel López S. ', 2; Mónica Troncoso Sch. '; María de los Angeles Avaria B.' 2, \\ Alvaro Clunes C. ${ }^{3}$; Marta Hernández Ch.'
}

Baclofen in spastic cerebral palsy

\begin{abstract}
Objetives to evaluole the effectiveriess pi boclofen versus flccebo to 'educe sogsticiv and improve molor 'unction. funct onal cutonomy, and self care. Design prospective two staces, tweek each, baclofen fi: to $2 \mathrm{ng} \cdot \mathrm{kg} \cdot \mathrm{cov}$ and placebo throrine. $37.5 \mathrm{mg}$ - d! cross over administret cn trial. Subjects: patients with spastc cerearal palsy living in a charily institution for invalid youngsters under medica ccre of a uriversity associoled public general hicspital pediatic neurclogy ol'nc at relropol ion Santiago. Chile. Twenky fersons agec 3 to 26 years with spastic cerebral pelsy. Measurenents and results: weekly assessments of squeletal muscle bone with the Ashworth scale iscored $:$ to 5 from normal througt. severe rigidityl showed signitizant reduction of spaslicity. from scores 3 or more in al palients at

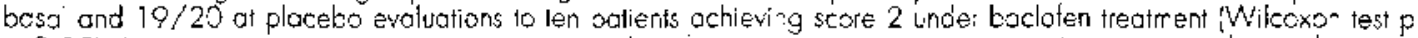
$<0.05$ i. Improvement in spontaneus moveme t and in routine ccre inanagerrent porticipolion wcs observed in nine patienls. Vo changes in molor furction were recoded. Grea improvement was seen in palie-ts with beller mieligence levels and less severe moto disfunctio-. Conclussion: boclofer should be considered a useful additional aid in the managerrent of patients with spaslic cereorel polsy. Furber stucies shoud be done to assess long term effecls of batofen on l'ese patients.
\end{abstract}

(Key words: cetebral salsy. spastic, bacloten !

La frecuencia de parálisis cerebral (PC) se estima entre 1,5 y $2,5 / 1000$ nacidos vivos' $y$ 1,2 a $2 / 1000$ en menores de 15 años ${ }^{2}$. Alrededor de $65 \%$ de estos pacientes sufren espasticidad, siendo ésta uno de los factores que produce mas alteraciones de la función motora y complicaciones musculoesqueléticas. En nuestro medio los trastornos motores, que incluyen espasticidad, dan cuenta de un tercio de las consultas a neurología pediátrica ${ }^{3}$. La rehabilitación de estos pacientes requiere considerables recursos, que se destinan de preferencia a fisioterapia, cuyos beneficios no han sido claramente demostrados $^{4-7}$; la cirugía ayuda a disminuir las limita-

I. Médico. Hospical Clínico San Borja Arriarán, Servicio de Neuropsiquiatria Infantil.

2. Depariamento de Pediatrín y Cirugia Infantil. Facultad de Medicina, Campus-Centro. Universidad de Chile.

3. Kinesiólogo. Pequeño Cottolengo Obra Don Orione. Financindo por el Departamento Técnico y de Investigación (DTI), Unipersidad de Chile. Proyecto (M3613). ciones de movimientos articulares y mejorar las posibilidades de deambulación de los niños afectados. No se ha dado suliciente relevancia al rol de fármacos antiespásticos como tratamiento adyuvante. Son tres los fármacos antiespásticos cuyo efecto ha sido demostrado en estudios controlados con doble ciego: dantroleno, diazepam y baclofeno $0^{8-11}$.

Dantroleno es una sal sódica hidratada, clasificada como un relajador muscular directo, que al mismo ticmpo reduce la fuerza muscular, por lo cual no puede espcrarse que mejore el control motor, limitándose su empleo a pacientes postrados. Cuando sc emplea por largo tiempo, puede causar hepatitis, crisis epilépticas o pericarditis. Diazepam actúa en la médula espinal facilitando la ncurotransmisión mediada por receptores para el neurotransmisor ácido gama amino butírico (GABA). Su uso es limitado porque causa somnolencia, sedación y dependencia.

Baclofeno (-(p-clorofenil)-GABA) es un mimético lipofílico del gama amino butirato, que actúa como relajador muscular de acción cen- 
tral, primariamente inhibiendo gamamotoneuronas y reflejos espinales monosinápticos y polisinápticos ${ }^{12}$. Aparte de su efecto antiespástico tiene propiedades analgésicas y reduce los problemas esfinterianos. Es bien tolerado, produce somnolencia pero comparativamente menos que diazepam. Su interrupción abrupta puede producir alucinaciones, convulsiones o diskinesias. Se ha ido ubicando como el antiespástico más usado, principalmente por vía intratecal, en adultos con espasticidad de origen medular ${ }^{\mathrm{j} \text { ? }}$. Las referencias a su empleo en espasticidad de origen cerebral se limitan a algunas en que la administración oral o intratecal produjo cambios positivos ${ }^{14-16}$.

Para evaluar los cfectos de baclofeno en la espasticidad debida a parálisis cerebral, se realizó un ensayo cruzado de administración oral đel medicamento con tiamina o vitamina B1, compuesto del cual no se han descrito electos sobre el tono muscular, a modo de placebo.

\section{Pacientes y Método}

El estudio sc realizó en 20 pacientes institucionalizados del Pequeño Coltolengo Don Orione, portadures de parálisis cerebral espástica de grado moderado o severo ${ }^{17}$ y tres o mís años de edad: que tuviesen márgenes de movilidad arricular sobre $50 \%$ en la mayoría de las articulaciones estudiadas; no hubjesen sido sometidos en Ios úlinios 6 meses a infiltraciones con fenol, cinugía o medicamentos destinados a modificar el tonn. la postura o controlar contracturas: no estuviesen recibiendo fátmacos sedanles que modifiquen el tono muscular y no tuviesen signos clínicos sugerentes de enfermedades progresivas en evoluciói. El júruitu mínimo de edad se estabiecio considerando que el trastorno motor tiene caráter evolutivo en la parailisis ccrebral, de modo que un diagnostico cierto sólo puede bacerse después de los dos años'. Además, cl examen ncurólogico es más uniforme y, por lo tanto, más comparab]e que en edades menores

El estudio incluía inicialmente 2 I sujeros. pero un varón fue excluido por sufrir frecuentes episodios intercurrentes de fiebre que obligaron a suspender los medicamentos, quedando la muesira constituida por 20 pacientes (1 l mujeres) cuva edad era $10,28 \pm 6,12$ años, márgenes de 3 y 26 años. Dieciséis de cllos sufrían tetraparesia espástica y cualro diptejia espástica. Quitce tenian intensa disminución de la motilidad espontánea y prácticamente carecían de control postural; cuatro lograban sedestacion y solameure una nifra era capaz de marchac independientemente. Diecisiete pacientes tenían retardo psiconotor scvero o profundo y tres moderado. Dicciséis sufrian epilepsia sintomática y estaban en ttatamiento con fárınacos anticonvulsivantes. La causa de la parálisis cerebral era presuntamente prenatal en ocho, perinatal en res. postnatal en cuatro casos, mientras que la historia chínica no aporta- ba antecedentes en los otrus cinco enfermos. Los padres o tutores de cada paciente fueron informados en detalle acerta de las características del estudio, solicitándoseles consentimiento escrito para participar en 61 .

Lus efectos de baclofeno oral (en comprimidos $10 \mathrm{mg}$ ) se compararon con los de tiamina (cn comprimidos de 25 Ing) de una manera cruzada. Los comprimidos de $25 \mathrm{mg}$ de tiamina o vitanina $B 1$ tienen la misma forma. tamaño y color que los de baclofeno, no parececen afectar el tono muscular. las reacciones de hipersensibilidad a ella son poco frecuentes $y$ empleada durante 6 semanas en dosis de $12.5 \mathrm{mg}$ cada 8 horas no tiene consecuencias adversas. $\mathrm{La}$ asiguación de la secuencia de medicamento y placebo y el esquema administración de los fúrmacos estuvo a cargo exclusivo $y$ en conocimiento de uno de los investigadores (MH). Diez pacientes fueron asigondos arbitrariamente a la secuencia bacloteno-tiamina y diez a liamina-baclofeno. Cada ensayo comprendió seis semanas. En la primera semana de administración te baclofeno se aumentó grodualmente la dosis a partir de $0,5 \mathrm{mg} \cdot \mathrm{kg}$ - día en tres fracciolies. hasta alcanzar una de inantención de 1 a $2 \mathrm{mg} \cdot \mathrm{kg} \cdot$ día. también en tres fracciones. Esta última dosis se manuvo durante cuatro semanas, para reducirla luego gradualmeste en la semana siguiente. La tiamina se administró en un esquema de medio comprimido tres veces al día (37.5 mg * dia). durantc an total de seis semanas. Ambas fases del ensayo se realizaron separadas por dos semanas en las que no se adıninistró ninguno de fos dos compuestos involucrados en el estudio.

Antes del ensayo se realizó a todos los pacientes un examen médico general y un cxamen neurológico. Ademís se realizó cvaluación cualitativa del tono muscular medionte la Escala de Ashworth ${ }^{18}$, instrumento que consta de cinco calegorías definidas (tabla $I$ ), de probada confiabilidad y reproducibilidad ${ }^{19}$. El tono muscular se evaluó en las articulaciones de codos. caderas, rodillas y tobillos. asignando calificaciones por separado a la flexión y la extensión, excluyendo articulaciones con deformidad evidente o linitación del desplazamicnto articular mayor a $\mathbf{5 0 \%}$. La calificacion de Ashworth fue aplicada por ires de los investigadores (IL. MT, AC), quienes, tras un período de entrenamiento, lograron una confiahijidad interexaminador de $82 \%$

El desempeño motor se midió mediante la Escala de Función Motora Grttesa 20, discfrada para detectar cambios de tsta en niřos con discapacidad física. Este instrumento

\section{Tabla 1}

Escala de calificación de espasticidad de Ashworth ${ }^{18}$

\begin{tabular}{|c|c|}
\hline Grado & Descripción \\
\hline 1 & No bay aumento de tono \\
\hline 2 & $\begin{array}{l}\text { Aumento leve del tono. Resistencia al flectar } \\
\text { o extender el segmento afectado. }\end{array}$ \\
\hline 3 & $\begin{array}{l}\text { Aumento moderado del tono. El segntento } \\
\text { afectido aún se flecta con facilidad }\end{array}$ \\
\hline 4 & $\begin{array}{l}\text { Aumento considerable de tono. Dificultad en } \\
\text { movimientos pasivos }\end{array}$ \\
\hline 5 & $\begin{array}{l}\text { Segmento afectado rígido en flexión y en ex- } \\
\text { tensión }\end{array}$ \\
\hline
\end{tabular}


incluye 8.5 aspectos de motricidad gruesa (cu posiciones supino, grono. con cuarro apoyos. setutado, de pie, caminando, escalando), que se clasifican en cuatro categorias de 0 (no puede iniciar) hasta 3 puntos ícompleta independientementel. con un total de 25.5. En este caso la escola se tradujo a actividades de la vida diaria (AVD), evaluando la capacidad y autonomía de los sujetos en alimentación. aseo, abrigo y movilización $y$ se aplić́ consultanda sobre ellas a las personas directamente encargadas del cuidado de ellos.

Durante todo el ensayo los pacientes fueron objeto de estrechos controles pediátricos y de cnfermería. que permitieron tegistrar enfermedades intercurrentes, convulsiones - efectos laterales atribuibles a los fámacos administrados. Las evalunciones se realizaron siempre en el mismo lugar y hora, procurando mantener la temperatura ambiente en alrededor de $20^{\circ} \mathrm{C}$. El tono muscular se midió en las semanas $2,3,4, y$ de cada ensayo. vale decit, ocho veces en cada paciente. La calidad de Ias actividades motota y de la vida diaria se midió en la semana 5 de casla fase del ensiyo.

En cada paciente se compararon los resultados obtenidos en la evaluación inicial, bajo baciofeno y placebo. En cada evaluación de tono muscular se calculó un puntaje glohal promediando las categorías obtcnidas en cadi auticulación. Luego se calculo el promedio de las cuatro mediciones efectuadas duante el tratamiento con baclofeno y las cuatro con placebo y se compararon los resulados de las dos fases mediante la prueba no paramétrica para diferencias de categorias de signo $t$ de Wilcoxon para los pares de evaluaciones efectuadas en cada semana, con el objeto de estimar el momento de mayor efeclo y para los proinedios de todos ellos en cada fase. Se comparó la proporción de pacientes en cada rango de la escala de Ashwirth en la mediciou inicial y en las dos fases dal ensayo y la proporción de los cambios observados con respecto a la basal inicial mediante la prueba de chi cuadrado. Tanbićn se analizo el efecto del orden de las fases del ensayo, cdad. intensidad del déficit motor. scveridad del retardo mental y dosis de baclofeno sobre los resultados obtenidos. Los efectos en la función motora gruesa se analizaron comparando el puntaje bruto obtenido en las tres evaluacinnes. con una prueba de $t$ pareado para diferencias de prome. dios. Para valorar los cambios en las actividades di vida se compararon las proporciones de pacientes que mostraban cambios en el grado de dependencia a de dificultad durante cacla fase con respecto a la busal.

\section{Resultados}

De 20 pacientes estudiados, tres sufrieron náuseas y vómitos asociados al aumento inicial de la dosis de baclofeno, debiendo prolongarse cl tiempo necesario para alcanzar las dosis definidas como terapéticas. No se registraron cambios en el estado general, aumento en la frecuencia de crisis o de enfermedades intercurrentes atribuibles a los fármacos durante las fases del ensayo o en los períodos de retirada de medicamentos.
Siete de 180 evaluaciones de tono muscular realizadas en diferentes pacientes fueron desechadas: tres en la fase de placebo y una en la de baclofeno debido a enfermedades intercurrentes que modificaban significativamente el estado del paciente y otras tres en la de baclofeno por no haberse alcanzado al momento de la primera evaluación una dosis de al menos $1 \mathrm{mg}$ - kg - día. La intensidad de la espasticidad disminuyó en un número significativamente mayor de pacientes en la fase de baclofeno que con tiamina (prueba t: $46, p<0,05$ ), encontrándose diferencias significativas entre las dos fases del cnsayo en las semanas 2 y 3 , pero no significalivas para las semanas 4 y 5 (tabla 2). La intensidad basal de la espasticidad era de grado 3 de la escala de Ashworth o mayor en todos los pacientes y en 19 de los 20 durante la fase de placcbo con tiamina, pero disminuyó significativamente en la de baclofeno, donde 10 de los 20 pacientes $(\mathrm{p}<0,01)$ mostraban espasticidad grado 2 o menor (tabla 3). El análisis de las variables edac, scveridad inicial de la espasticidad y secuencia de ensayos no mostró influencias significativas en relación al efecto del tratamiento. No se observaron diferencias entre dosis de 1 y $2 \mathrm{mg} \cdot \mathrm{kg} \cdot$ día, hecha la salvedad que los tres casos en que no se alcanzaron dosis dc al monos $1 \mathrm{mg} \cdot \mathbf{k g} \cdot$ día durante la segunda semana de la fase de baclofeno no fueron considerados en el análisis correspondiente. Los tres niños con menor alteración del átea cognitiva mostraban inicialmente espasticidad de grado mayor de 3 y cn algunos segmentos de grado 4.

\section{Tabla 2}

Comparación del cambio en la espasticidad (t) entre las fases de tratamiento con placebo y baclofeno en las semanas 2 a 5 de cada ensayo

\begin{tabular}{cccc}
\hline Semana & $\boldsymbol{n}^{*}$ & $\mathbf{t}$ & $\mathbf{p}$ \\
\hline 2 & 15 & 22 & $<0.05$ \\
3 & 20 & 48 & $<0.05$ \\
4 & 19 & 55 & $\mathrm{~ns}$ \\
5 & 19 & 52 & $\mathrm{~ns}$ \\
\hline Total & 20 & 46 & $<0,05$ \\
\hline
\end{tabular}

* n varía por exclusión de siete evaluaciones de tono (ver texto).

p: prueba de Wilcoxon para diferencias de rango. 
Tabla 3

Distribución de los pacientes segün grado de espasticidad (Escala de Ashworth) en la evaluación inicial y en las fases de rsatamiento con placebo y baclo[ent]

\begin{tabular}{cccc}
\hline $\begin{array}{c}\text { Ashworth* } \\
\text { Grados }\end{array}$ & $\begin{array}{c}\text { Basal } \\
\text { (n) }\end{array}$ & $\begin{array}{c}\text { Placebo } \\
\text { (n) }\end{array}$ & $\begin{array}{c}\text { Baclofeno } \\
\text { (n) }\end{array}$ \\
\hline 1 & 0 & 0 & 0 \\
2 & 0 & 1 & 10 \\
3 & 9 & 11 & 9 \\
4 & 10 & 8 & 1 \\
5 & 1 & 0 & 0 \\
\hline
\end{tabular}

* promedio de los segmentos examinados

Basal / Placebo : X2: $2.4 \mathrm{~ns}$

Basal /Baclofeno : X2: $18.4 \mathrm{p}<0,01$

Placebof Baclofeno : X2: $13.0 p<0,01$

junto con intenso aumento de tono y diffeultades en las movilidades pasiva y activa: todos se beneficiaron durante la fase de baclofeno con cambios que los llevaron a un grado de 2 o leve resistencia del segmento afectado.

El desempeño en función motora estaba severamente disminuido en todas las evaluaciones basales, con calificaciones de 35,5 $\pm 47,1$ para el conjunto de los pacientes, cercano a $13 \%$ de la calificación total de la escala y a $17 \%$ de lo esperado en un niño normal de 3 años. Sólo cr una paciente, con diplejia espástica moderada, fuc posible aplicar todos los ítemes de la escala. En ringuna de las fases del ensayo se observó cambios en la calificación promedio del grupo, pero en tres casos de inenor compromiso cognitivo y mayor control motor que el promedio (EI: 54, 66, 212) se registraron aumentos de 27,14 y 11 puntos en la fase de baclofeno, que se reftejo. en uno por inicio de marcha con apoyo, en otro por mejor control del tronco y en marcha algo más expedita en la tercera paciente.

Se observaron cambios favorables en actividades de vida diaria en $9 / 20$ pacientes durante la fase con baclofeno y en $3 / 20$ durante la de placebo, coincidiendo con aquellos en que disminuyó la espasticidad. Los cambios consistieron en mayor facilidad para estirarse y durante los cambios de ropa, más movilidad espontánea y en una niña aumento de la autonomía para alimentarse.

\section{Comentario}

Los ensayos abiertos con antiespásticos son difíciles por la subjetividad de las apreciaciones de evaluadores y padres ${ }^{14}$. Para observaciones más objetivas los estudios deben ser ciegos y los instrumentos y procedimientos estándares. La movilización pasiva repetida de los segmentos corporales, la temperatura ambiente, las emociones y las condiciones generales producen cambios del tono muscular en los pacientes espásticos, por lo que deben ser uniformes en el momento de la evaluación. Las posiciones en que se evalúa cada segmento también deben ser estánđar, ya que modifican la percepción de la resistencia a la movilidad ${ }^{14.21}$.

Los resultados obtenidos en este ensayo muestran un efecto favorable de baclofeno sobre la espasticidad, en concordancia a lo reportado por otros autores ${ }^{14.15}$. El número basal de 11 pacientes con espasticidad de categoría 40 mâs se redujo a sólo uno en la fase de baclofeno, versus ocho en fase de placebo, con un traspaso paralelo de pacientes a la categoría 2 , de menor espasticidad durante la administración del medicamento. La magnitud del cambio producido por el baclofeno es también mayor que con placebo. Si bien en otras experiencias se han obtenido resuitados aun más acentuados, éstos pueden atribuirse en parte a la severidad de las alteraciones de nuestros pacicntes y a la condición de institucionalización. Se ha sugerido ${ }^{14}$ que la menor edad podtía ser un factor favorable, lo que no comprobamos en nuestra serie, tal vez por las características de la muestra. En cambio el menor daño intelectual y motor parecen favorecer un efecto útil de los antiespásticos. La ausencia de diferencias en los efectos de baclofeno a dosis entre J y 2 Ing $-k$ - día sugiere que en futuros estudios pudiese ser conveniente titular éstas en niveles mayores. Cabe señalar que en los pacientes que recibían dosis menores a $1 \mathrm{mg} \cdot \mathrm{kg}$ • día en la primera evaluación. por presentar efectos laterales, no se registraron cambios en la magnitud de la espasticidad o en las actividades de vida diaria.

La ausencia de cambios significativos en el control motor puede deberse a diferentes factores. En primer término, la severa discapacidad mental y motora de los pacientes estudiados, la mayoría de ellos no funcionales y dependientes 
de otra persona para satisfacer necesidades bâsicas. En segundo término, la condición de vivir en una institución de caridad que no dispone de un sistema de rehabilitación continua, a lo que posiblemente se agrega un componente de $\mathrm{pri}^{\mathrm{i}}$ vación afectiva. La brevedad del seguimiento y la naturaleza del ensayo, diseñado para evaluar un efecto inmediato, principalmente sobre $\mathrm{e}]$ tono muscular, no favorecen la consecución de objetivos de mayor plazo y que requerirían intervenciones más integrales con resultados más lardíos, como serían los cambios en el contro] motor, a lo que hacen excepción los pacientes con monor deterioro motriz e intelectual. Los beneficios para las actividades de la vida diaria, si bien modestos, representan para estos pacientes una mejoria significativa en su calidad de vida, cumpliéndose así con uno de los objetivos planteados.

El uso de fármacos como coadyuvantes en el manejo de los niños con parálisis cerebral tienc como principal objetivo mejorar la calidad de vida de los pacientes. aliviando las molestias derivadas de la espasticidad, y las habilidades motoras en tanto éstas son vía de acceso a la exploración del medio y a ta funeionalidad. Asî, pues, mientras en algunos casos es conveniente reducir la espasticidad excesiva para evitar posiciones forzadas, mejorar la movilidad o facilitar los cuidados del paciente, en otros la alteración es un elemento favorable, como en muchos en que permite la bipedestacion y la marcha: cn ellos no está indicado tratar de disminuirla con fármacos $u$ otros medios ${ }^{14}$.

La administración intratecal de baclofenn en pacientes con parálisis cerebral produce disminución del tono muscular, aunque preferentcmente de extremidades inferiores ${ }^{16}$, y su cmpleo continuo y prolongado reduce la espasticidad de causa medular y mejora la funcionalidad de estos pacientes, pero es un procedimiento caro y con frecuentes complicaciones ${ }^{22.23}$. No conocemos estudios que exploren el efecto de tratamientos orales a largo plazo con antiespásticos, sin embargo, si se dernuestran efectos positivos sobre el tono muscular en períndos cortos, es posible que pudiesen tener utilidad en la prevención de deformidades y retracciones. Nuestros resultados sugieren que el baclofeno oral puede ser útil como parte del tratamiento de pacientes con espasticidad de origen central y estimulan a continuar en la evaluación comparati- $\mathrm{va}$, con otras vías de administración ${ }^{16}$, agentes $\mathrm{y}$ procedimientos destinados a disminuir la espasticidad 24.25 .

\section{Resumen}

Propósito: comparar la efectividad de baclofeno y un placebo para disminuir la espasticidad, mejorar la función motora, autonomía funcional y participación en sus cuidados diarios. Diseño: prospectivo, cruzado, de administración de baclofeno (1 a $2 \mathrm{mg} \cdot \mathbf{k g} \cdot$ día) y placebo (tiamina, $37.5 \mathrm{mg} \cdot$ día) en dos fases de seis scmanas cada una. Situación: pacientes con parálisis cerebral de una institución privada para e] cuidado de inválidos, bajo control y tratamiento en la unidad de neurología pediátrica del Hospital Clínico San Borja-Artiarán, en Santiagn metropolitano. Veinte personas de 3 a 26 años de edad, con parálisis cerebral espástica. Mediciones y resultados: en la cvaluación inicial del tono muscular con la escala de uno (normal) a cinco puntos (rigidez severa) de Ashworth, todos los pacientes tenían puntajes de espasticidad iguales o mayores a 3 , lo que no cambió bajo placebo, pero disminuyó significativamente durantc el tratamiento con baclofeno, en que el tono bajó en 10 casos a puntaje 2 (prueba de Wilcoxon $p<0,05)$. En nucve pacientes mejoraron lat movilidad espontánea y el desempeño cn sus cuidados de rutina en la fase de baclofeno. No se registraron cambios en la función motora gruesa. Los beneficios fueron mayores en los pacientes con menores deterioros intelectual y motor, en todos los aspectos. El baclofeno puede ser un tratamiento auxiliar útil en pacientes con parálisis cerebral espástica y merece ser evaluado cn ensayos de mayor duración.

(Palabras clave: parálisis ccrebral espástica, baclofeno.)

\section{Agradecimientos}

A los pacientes y sus familias. Al personal, profesinnales y religiosos del Pequeño Cottolengo Obra Don Orione. Cerrillos. A Laboratorios Ciba-Geigy Chile, que suministró los medicamentos.

\section{Referencias}

1 Kuban KCK. Leviton A: Cerebrat palsy. New Engl J Med 1994; 330; 188-195. 
2. Neisun K. Suriman K. Russmen B: Cerebral Halsy. In: Pediatric Neurology. ed. por KF Swaiman, MosbyYear book Inc., 1994: 47I-488,

3. Novoa F. Avaria MA. Trnucoso L: El uso del riempo en un policlínico de Neurolngía. VIII Reunión Anual de la Sociedad de Psiquiatria y Neurologia de la Infancia y Adolescencia. Santiago, 1990.

4. Prine RS: On the treatment of cerebral palsy. The outcome of 177 patients. 74 totally untreated. Pediatrics 1962: 29: 605-607.

5. Wright T, Nicholson J: Physiotherapy for the spastic child: An evaluation. Dev Med Child Neurol I973; 1.5: 146-163.

6. Palmer FB, Shapiro BK, Watchel RC, et at: The effects of physical therapy on cerebral palsy. New Engt J Med 1988; 318: 803-808.

7. Dunkeld Tarmbul] d: Early intervention for children with or at risk of cerebral palsy. Am J Dis Child 1992; 147: $54-59$

8. Young R. Defiraide PJ: Spasticity (first of two parts) New Eng] J Med 1981: 304: 28-33

9. Youns R. Deluride PJ: Spasticily (second of two parts). New Engl J Med 198 I; 304: 96-99.

10. Daridoff RA: Antispasticity drugs: Mechanisnus of action. Ann Neurol 1985; 17: 107-1]6.

11. Deluside $P$ : Oral treatment with current muscle relaxants. In: Treating spasticty: pharmacological advances. Ed. por David Marsden. Hans Huber Publishers, 1989: 31-37.

12. Bimiger $H$ : Basic aspects of inhibitory transmilier aminoacids in spasticity and pain. In: Treating spasticity: pharmacological advances. EJ. por David Marsden. Hans Huber Publishers, 1989: 20-30.

13. Armstrous $R$ : Intrathecal baclofen and spasticity: What do we know and what dowe need to know. Dev Med Child Neurol 1992: 34: 739-745.
14. MiHa PJ, Jaksam ADM: A controlled Irial of baclofen it children with cerebral palsy. I Int Med Res 1977; 5: 398-404.

15. Milemov IG: Mechanisms of haclofen action on spaticiry. Acta Neurol Scand 1992; 85: 305-310.

16. Athright AL. Cervi A, Singletay J: Intrathecal baclofen for spasticity in cerebral palsy. JAMA 1991: 265: 1418-1422.

17. Minear WL: A classification of cerebral palsy. Pediatrics 1956; 18: 841-842.

18. Arhworth B: Preliminacy trial of carisoprodol in multiple sclerosis. Practitioner 1964; 192: $540-542$.

19. Kin Chus Lee: The Ashworth seale. A reliable and reproducible method of measuring spasticity. Neurology 1989:39: J43.

20. Rusself D.f. Rosenbaum PL, Cadman DT, Grwhand C. Hardy $S$, Jtrits $S$ : The gross motor function measure a means to cvaluate the elfects of physical therapy. Dev Med Child Neurol 1989:31: 341-352.

21. Price R. Lehmun JF, Boswell-Bessefte Sh, Burleigh A de Lireur $B J$ : Influence of cryotherapy on spasticity at the heınan ankle. Arch Phys Med Rehabil 1993; 74: $300-304$.

22. Ochs $G$. Struppler A. Meyerson BA, el al: Intrathecal baclofen for long-tern treatment of spascicity: a muiticentre studt. Journal of Neurology. Neurosutgery and Psychiatry 1989; 52: $933-939$.

23. Meyrwer JM, Sreers WD, Tuel SM, Cross LL Howerth CS: Continuous intrathecal baclofen in spinal cord spasticity, Ain J Phys Mcd Rehabil 1992: 71: 321-327.

24. Soldifurd PR. Spenyles SE. Sawasky KB. Clonidine in the treatment of brainstem spasticity. Am J Phys Med Rehabil 1992; 71: 301-303.

25. Calderin-Cronzrilez $k$, Cudterim-Sepinledit $R$. RinconReyes M. Gatrin-Ranirez J. Mino-Arango E: Botulinum toxin $A$ in management of cerebral palsy. Pediatr Neurol 1994: 10:284-288. 